\title{
Biological Characteristics and Genetic Diversity of Phomopsis asparagi, Causal Agent of Asparagus Stem Blight
}

\author{
Y. Q. Yang, ${ }^{1}$ Q. Sun, ${ }^{2}$ C. M. Li, ${ }^{3}$ H. F. Chen, ${ }^{1}$ F. Zhao, ${ }^{2}$ J. H. Huang, ${ }^{1}$ J. S. Zhou, ${ }^{4}$ X. M. Li, ${ }^{1, \dagger}$ and B. Lan ${ }^{1, \dagger}$ \\ ${ }^{1}$ Institute of Plant Protection, Jiangxi Academy of Agricultural Sciences, Nanchang 330200, China \\ ${ }^{2}$ Huangdao Customs House, Qingdao 266555, China \\ ${ }^{3}$ Jiangsu Lixiahe Institute of Agriculture Science, Yangzhou 225007, China \\ ${ }^{4}$ Institute of Vegetables and Flowers, Jiangxi Academy of Agricultural Sciences, Nanchang 330200, China
}

\begin{abstract}
Asparagus stem blight is a regional disease. In the present study, we compared strains of Phomopsis asparagi from six different provinces to determine their biological characteristics and genetic diversity, differences in the pycnidium and conidium production, pathogenicity, and growth rate. Considerable differences were established in the pycnidium and conidium production among the $P$. asparagi strains from the six studied provinces. The largest pycnidium and conidium production had the strains from Fujian, followed by those from Hainan. The virulence of $P$. asparagi strains was significantly different but without a correlation with the geographical source of the strain. FJ2 had the highest virulence, followed by HN2, SD4, and SD5, whereas SD5 had the lowest virulence. The colony diameter and dry weight of the strains of asparagus stem blight fungus from the six provinces were substantially different. The

sequence repeat (ISSR) primers. ISSR-PCR amplification was performed on 36 strains of asparagus stem blight fungus, and a large number of repeatable DNA fingerprints were obtained. Most of the amplified fragments were within 300 to $500 \mathrm{bp}$. In all, 69 total points, 64 multiple points, and $92.75 \%$ polymorphism points were established. The number of ISSR gene sites detected by four primers ranged from 14 to 20, with an average of 16 multiple sites. The copolymerization was divided into three groups: XT1-5, LT1-3, and FJ1-5, which were clustered into the first group; SD1-6, SX1-6, and HB1-6, clustered into the second group; and HN1-5 in the third group. The results of the cluster analysis revealed that the strains of the neighboring provinces had a nearer phylogenetic relationship than that between distant ones. Therefore, the system evolution of $P$. asparagi is related to the geographical distribution of its strains.
\end{abstract} colonies of HN1-5 had the largest diameters, whereas those of XT1-5, LT1-3, FJ1-5, and SX6 had smaller diameters. Four primers with good repeatability and strong specificity were selected from 100 intersimple
Keywords: asparagus stem blight; biological characteristics; genetic diversity; ISSR-PCR; $P$. asparagi
Asparagus officinalis Linn belongs to the family Liliaceae, genus Asparagus. It is one of the 10 most famous dishes in the world, known as the "king of vegetables" in the international market. It is exceedingly popular and loved by people for its high nutritional value and abundant health benefits such as lung moistening, cough relief, phlegm elimination, tumor growth inhibition, and so on (Chen 2005; Liu 2001; Liu et al. 1994).

In recent years, the occurrence of asparagus diseases has been continually increasing along with the expansion of its cultivated area in the world. The occurrence and harm of stem blight disease has seriously affected asparagus yield and quality (Liu et al. 2008; Meng et al. 2013; Yang et al. 2012a). The pathogen of asparagus stem blight which causes this globally distributed devastating disease is Phomopsis asparagi (Sacc.) Grove (Davis 2001; Liu et al. 1991; Miao et al. 1991; Uecker and Johnson 1991; Yang et al. 2012b, 2015a). The most serious damage has been established in China, Japan, Thailand, Indonesia, and other

${ }^{\dagger}$ Corresponding authors: X. M. Li, xmli@aliyun.com; and B. Lan, lanbo611@163.com

Y. Q. Yang, Q. Sun, and C. M. Li contributed equally to this work.

Funding: This research was supported by the National Natural Science Foundation of China (grant number 31460456), Special Fund for Technology Innovation Guide Engineering of Jiangxi (grant number 20192AEI91009), Jiangxi Talent Support Program for Distinguished Young Scholars (grant number 20171BCB23081), Key Projects of Science and Technology in Jiangxi (grant number 20151BBF60067), and Natural Science Foundation of Jiangxi (grant number 20202ACBL205006 and 20142BAB214021).

The author(s) declare no conflict of interest.

Accepted for publication 6 May 2020.

(C) 2020 The American Phytopathological Society
Asian asparagus-growing countries. In China, this disease occurs widely but is more severe in the southern part of the country than in the northern. Even slight damage of produce decreases its production rates and quality; the diseased plants die prematurely in severely damaged fields, destroying the whole production (Yang et al. 2015b, 2016, 2018). Hot and humid weather conditions are favorable for the occurrence of the stem blight disease. However, the main asparagus production areas in Europe and the United States are characterized by cold and cool climate. Thus, stem blight occurrence in Europe and the United States is minor, and there are fewer relevant reports from studies conducted in these regions (Davis 2001; Elena 2006; Sonoda et al. 1997; Uecker and Johnson 1991).

Biological characteristics are an important trait of plant fungi, and strains from different geographical sources often show specific differences in their biology and genetics (Yang et al. 2012b). The differences in the biological characteristics and virulence of $P$. asparagi strains in Fujian, Zhejiang, Jiangxi, Hubei, Jiangsu, and Shandong Provinces were previously compared (Liu et al. 1994). The biological characteristics of the strains from Jiangxi, Fujian, Hebei, Shandong, Hainan, and other provinces were compared in a previous study, and the phylogenetic relationship of these strains was analyzed based on ribosomal DNA (rDNA) internal transcribed spacer (ITS) (Yang et al. 2012b).

The intersimple sequence repeat (ISSR) marking method is a new molecular marking technique developed in microsatellite technology in recent years (Guleria et al. 2007; Reddy et al. 2002). ISSR technology can detect the polymorphism of genomic DNA rapidly, efficiently, and sensitively; has good repeatability, simple operation, and low cost; and is suitable for the detection of large samples. It has been applied rapidly in population genetics, germplasm resources, taxonomy, and phylogenetics. Many scholars studied the genetic diversity of plant fungi using the ISSR marking method (Guo et al. 2017; Karwasra et al. 2002; Sharma et al. 2005; Stodart et al. 2007). However, to our knowledge, there have been no similar reports for $P$. asparagi.

China has a vast territory, with a distance of more than $5,500 \mathrm{~km}$ between the north and the south and a span of about $50^{\circ}$ of latitude; because of this, it has abundant and changeable climatic resources. In 
this study, 36 strains from three climatic zones were picked to explore biological characteristics and genetic diversity (Fig. 1). Shandong, Shanxi, and Hebei Provinces belong to the north temperate zone of China. Jiangxi and Fujian Provinces belong to the subtropical zone of China. Hainan Province belongs to the tropical zone of China. The biological characteristics and genetic diversity of $P$. asparagi strains from Jiangxi, Fujian, Hebei, Shandong, Shanxi, and Hainan Provinces have been studied. This study attempted to establish whether there exists regional differentiation among $P$. asparagi strains, how the regional differentiation performs, and which factors influence the evolution of $P$. asparagi from different regions.

\section{Materials and Methods}

Materials. A. officinalis germplasm was obtained from the Creative Center of A. officinalis in Jiangxi Academy of Agricultural Sciences. The asparagus stem blight pathogen of $P$. asparagi strains XT1-5 and LT1-3 from Jiangxi Province, FJ1-5 from Fujian Province, HN1-5 from Hainan Province, SD1-6 from Shandong Province, SX1-6 from Shanxi Province, and HB1-6 from Hebei Province were conserved by our laboratory. All of the above strains were identified by morphology and molecular biology analyses and confirmed to be $P$. asparagi (Yang et al. 2012b). The details of the strains are presented in Table 1. Potato dextrose agar (PDA) and Luria-Bertani cultures were prepared as described earlier by Fang (1998). Detailed information, including the origins of 36 strains of $P$. asparagi, is provided in Table 1.

Differential analysis of pycnidium and conidium productions. A volume of $10 \mathrm{ml}$ of PDA medium was poured into a 9-cm-diameter Petri disk. Then, the disk with PDA medium was placed on the asepsis work table for $5 \mathrm{~min}$ to dry out the surface of the medium. Next, mycelium plugs were made using a 5-mm-diameter hole puncher, and one plug was transferred to a fresh PDA plate and cultured at $25^{\circ} \mathrm{C}$ and whole-day light (Yang et al. 2015a). The pycnidium numbers from different provinces were compared after 14 days. The spores in the Petri dish were scraped off with a glass carrier, wrapped in gauze, and placed in a glass mortar (with a diameter of $9 \mathrm{~cm}$ ), followed by the addition of $10 \mathrm{ml}$ of sterile water and milling with a pestle until the spores were released (Yang et al. 2012b). After filtration, the conidium concentration was determined using a blood cell count board, and the number of the pycnidia in each dish was counted (Yang et al. 2015a). Six replicates were performed for each strain.

Analysis on difference of pathogenicity to different strains. Seven grading standards for inoculation at the seedling stage were

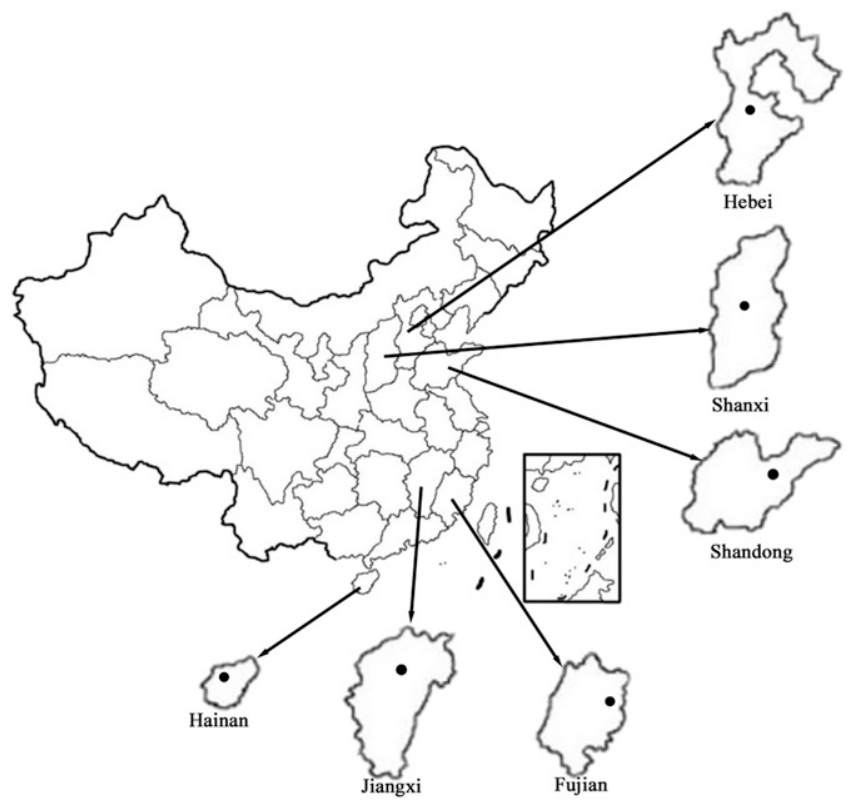

Fig. 1. Origins of 36 isolates of Phomopsis asparagi collected from six provinces in China. Black dots show the detailed location of the isolates from each province. formulated. The detailed grading standards are presented in Table 2. Twenty young (1-month-old) asparagus seedlings with a plant height of 15 to $20 \mathrm{~cm}$ were selected. Each plant was first placed in a round seedling pot with a diameter of $8 \mathrm{~cm}$, which was then put into a 30-by-50-cm plastic plate. Spore suspensions were prepared from the strains of the different provinces to reach a concentration of $1 \times 10^{6}$ spores $/ \mathrm{ml}$, and sprayed with Tween-20 (final concentration $0.02 \%$ ). The seedlings were sprayed with the spore suspension and kept in the dark at 25 to $28^{\circ} \mathrm{C}$ for 2 days, then transferred into a greenhouse at 25 to $28^{\circ} \mathrm{C}$; a humidifier was used to maintain the relative humidity at 90 to $100 \%$. Eight days later, we performed observations and recorded the results of the test. The test was repeated three times, each of which included 20 asparagus seedlings, and the grade of 20 asparagus seedlings was investigated to calculate the disease index. The grading standards of asparagus stem blight are provided in Table 2.

Comparison of growth rate among different strains. Mycelium plugs were made using a 5-mm-diameter hole puncher, and one plug was transferred to a fresh PDA plate and cultivated at $25^{\circ} \mathrm{C}$ for 8 days. It was centered on the mycelium block in the Petri dish and its colony diameter was measured. The 5-mm-diameter mycelium block of $P$. asparagi was attached to the PDA plate and cultivated at $25^{\circ} \mathrm{C}$ for 5 days; then, the 5 -mm-diameter hole puncher was used, and one mycelium block was transferred to a triangle bottle containing $50 \mathrm{ml}$ of potato dextrose broth culture solution at $25^{\circ} \mathrm{C}$, and shaker oscillation culture at $120 \mathrm{rpm}$ was implemented for 8 days. Next, the mycelia were filtered by a vacuum pump using preweighed filter paper, then put together with the filter paper and dried for 8 days in a drying oven at $50^{\circ} \mathrm{C}$. The sample was taken out for weighting and we calculated the average value. Six replicates were performed for each strain.

Table 1. Strains of Phomopsis asparagi used in this study and their origins

\begin{tabular}{llcc}
\hline Strain code & Sampling locality & Sampling date in 2011 & Sample size \\
\hline XT1-5 & Nanchang, Jiangxi & 19 June & 5 \\
LT1-3 & Nanchang, Jiangxi & 8 July & 3 \\
FJ1-5 & Fuzhou, Fujian & 25 June & 5 \\
HN1-5 & Danzhou, Hainan & 18 July & 5 \\
SD1-6 & Weifang, Shandong & 29 July & 6 \\
SX1-6 & Taiyuan, Shanxi & 9 August & 6 \\
HB1-6 & Baoding, Hebei & 19 August & 6 \\
\hline
\end{tabular}

Table 2. Grading standards of asparagus stem blight

\begin{tabular}{|c|c|}
\hline Disease grades & Symptom description \\
\hline 0 & No symptom \\
\hline 1 & $\begin{array}{l}\text { Diseased area } \leq 10 \% \text { of the area of main stem } \\
\text { and lateral branch of crown plant }\end{array}$ \\
\hline 3 & $\begin{array}{l}10 \% \text { of the area of main stem and lateral } \\
\text { branch of crown plant }<\text { diseased area } \leq \\
30 \% \text { of the area of main stem and lateral } \\
\text { branch of crown plant }\end{array}$ \\
\hline 5 & $\begin{array}{l}30 \% \text { of the area of main stem and lateral } \\
\text { branch of crown plant }<\text { diseased area } \leq \\
50 \% \text { of the area of main stem and lateral } \\
\text { branch of crown plant }\end{array}$ \\
\hline 7 & $\begin{array}{l}\text { Diseased area }>50 \% \text { of the area of main stem } \\
\text { and lateral branch of crown plant }\end{array}$ \\
\hline
\end{tabular}

Table 3. Intersimple sequence repeat primers and their sequences used in this study

\begin{tabular}{llc}
\hline Primer & Sequence $\left(\mathbf{5}^{\prime} \rightarrow \mathbf{3}^{\prime}\right)$ & Nucleotide count \\
\hline 861 & ACCACCACCACCACCACC & 18 \\
888 & BDBCACACACACACACA & 17 \\
889 & DBDACACACACACACAC & 17 \\
\hline
\end{tabular}


Statistical analysis. All the data were statistically analyzed using SAS 9.0 software through the Duncan's new complex range method.

ISSR genetic analysis. Using the culture method of a cellophanePDA plate, 36 strains were cultured at $25^{\circ} \mathrm{C}$ in the dark. After 3 days, the mycelium on the glass paper was shaved off and collected with a scalpel. After vacuum drying, it was transferred into a $1.5-\mathrm{ml}$ centrifuge tube and placed for storage in a refrigerator at $-20^{\circ} \mathrm{C}$. DNA extraction was carried out by the method reported by Rosewich et al. (1999).

In total, 12 strains were randomly selected (2 strains from each province) to screen the 100 ISSR primers (published by Columbia University in 2008). Four primers, synthesized by Beijing Aoke Biology Company (Beijing, China), with clear and repeatable strips were selected (Table 3). In this study, ISSR-PCR amplification with these four primers was used to investigate 36 asparagus stem blight strains, and 1 rice blast fungus Pyricularia oryzae was utilized as an external control strain. A preliminary experiment was carried out to optimize the PCR system, and the optimized PCR system is as follows. The total volume of the reaction was $25 \mu \mathrm{l}$, and the reaction mixture consisted of $2.5 \mu \mathrm{l}$ of $10 \times$ PCR buffer, $2 \mu \mathrm{l}$ of dNTP mix $(2.5 \mathrm{mM}), 0.2 \mu \mathrm{l}$ of Taqase ( $5 \mathrm{U} / \mu \mathrm{l}), 1 \mu \mathrm{l}$ of the primer $(0.5 \mu \mathrm{mol} / \mathrm{liter}), 1 \mu \mathrm{l}$ of DNA template (approximately $8 \mathrm{ng}$ ), and $18.5 \mu \mathrm{l}$ of double-distilled $\mathrm{H}_{2} \mathrm{O}$. The amplification program included the following steps: prevariant for $4 \mathrm{~min}$ at $94^{\circ} \mathrm{C} ; 38$ cycles of denaturation for $1 \mathrm{~min}$ at $94^{\circ} \mathrm{C}$, annealing for $1 \mathrm{~min}$ at $56^{\circ} \mathrm{C}$, and extension for $1 \mathrm{~min}$ at $72^{\circ} \mathrm{C}$; and, finally, extension for $10 \mathrm{~min}$ at $72^{\circ} \mathrm{C}$. The amplification product was detected by electrophoresis on a $1 \%$ agarose gel with Goodview, and the

Table 4. Pycnidium and conidium productions of Phomopsis asparagi strains $^{\mathrm{z}}$

\begin{tabular}{|c|c|c|}
\hline Strains & $\begin{array}{l}\text { Pycnidium production } \\
\text { (per Petri disk) }\end{array}$ & $\begin{array}{l}\text { Conidium production } \\
\text { (odu }{ }^{6} \text { per Petri disk) }\end{array}$ \\
\hline XT1 & $12.67 \pm 4.19 \mathrm{~h}$ & $34.50 \pm 4.19 \mathrm{e}$ \\
\hline XT2 & $15.83 \pm 3.60 \mathrm{~h}$ & $33.50 \pm 3.60 \mathrm{e}$ \\
\hline XT3 & $15.50 \pm 2.27 \mathrm{~h}$ & $30.83 \pm 2.27 \mathrm{e}$ \\
\hline XT4 & $17.33 \pm 3.63 \mathrm{~h}$ & $38.17 \pm 3.63 \mathrm{e}$ \\
\hline XT5 & $15.33 \pm 3.44 \mathrm{~h}$ & $36.33 \pm 3.44 \mathrm{e}$ \\
\hline LT1 & $20.50 \pm 4.11 \mathrm{hg}$ & $40.50 \pm 4.11 \mathrm{e}$ \\
\hline LT2 & $16.83 \pm 3.75 \mathrm{~h}$ & $35.83 \pm 3.75 \mathrm{e}$ \\
\hline LT3 & $16.67 \pm 2.23 \mathrm{~h}$ & $32.50 \pm 2.23 \mathrm{e}$ \\
\hline FJ1 & $62.50 \pm 5.56 \mathrm{a}$ & $203.33 \pm 5.56 \mathrm{~b}$ \\
\hline $\mathrm{FJ} 2$ & $47.00 \pm 7.68 \mathrm{bdc}$ & $191.00 \pm 7.68 \mathrm{cb}$ \\
\hline FJ3 & $55.67 \pm 6.00 \mathrm{ba}$ & $197.33 \pm 6.00 \mathrm{cb}$ \\
\hline FJ4 & $60.17 \pm 5.66 \mathrm{a}$ & $188.67 \pm 5.66 \mathrm{c}$ \\
\hline FJ5 & $51.33 \pm 7.17 \mathrm{bac}$ & $216.67 \pm 7.17 \mathrm{a}$ \\
\hline HN1 & $39.67 \pm 7.62 \mathrm{edf}$ & $110.00 \pm 7.62 \mathrm{~d}$ \\
\hline $\mathrm{HN} 2$ & $34.50 \pm 5.39$ ef & $104.5 \pm 5.39 \mathrm{~d}$ \\
\hline HN3 & $38.67 \pm 5.94$ edf & $101.33 \pm 5.94 \mathrm{~d}$ \\
\hline HN4 & $43.17 \pm 5.30 \mathrm{edc}$ & $105.67 \pm 5.30 \mathrm{~d}$ \\
\hline HN5 & $31.00 \pm 6.71 \mathrm{gf}$ & $99.67 \pm 6.71 \mathrm{~d}$ \\
\hline SX1 & $13.83 \pm 2.70 \mathrm{~h}$ & $32.17 \pm 2.70 \mathrm{e}$ \\
\hline SX2 & $14.17 \pm 2.79 \mathrm{~h}$ & $27.50 \pm 2.79 \mathrm{e}$ \\
\hline SX3 & $15.33 \pm 4.43 \mathrm{~h}$ & $30.83 \pm 4.43 \mathrm{e}$ \\
\hline SX4 & $11.33 \pm 2.69 \mathrm{~h}$ & $31.17 \pm 2.69 \mathrm{e}$ \\
\hline SX5 & $12.50 \pm 3.16 \mathrm{~h}$ & $33.67 \pm 3.16 \mathrm{e}$ \\
\hline SX6 & $12.67 \pm 2.42 \mathrm{~h}$ & $34.33 \pm 2.42 \mathrm{e}$ \\
\hline SD1 & $14.00 \pm 2.46 \mathrm{~h}$ & $33.83 \pm 2.46 \mathrm{e}$ \\
\hline SD2 & $13.83 \pm 3.42 \mathrm{~h}$ & $30.17 \pm 3.42 \mathrm{e}$ \\
\hline SD3 & $16.17 \pm 2.51 \mathrm{~h}$ & $33.33 \pm 2.51 \mathrm{e}$ \\
\hline SD4 & $15.83 \pm 3.50 \mathrm{~h}$ & $36.50 \pm 3.50 \mathrm{e}$ \\
\hline SD5 & $14.83 \pm 3.41 \mathrm{~h}$ & $29.33 \pm 3.41 \mathrm{e}$ \\
\hline SD6 & $14.17 \pm 3.69 \mathrm{~h}$ & $30.17 \pm 3.69 \mathrm{e}$ \\
\hline HB1 & $13.83 \pm 3.19 \mathrm{~h}$ & $34.00 \pm 1.95 \mathrm{e}$ \\
\hline HB2 & $14.17 \pm 1.89 \mathrm{~h}$ & $31.67 \pm 3.52 \mathrm{e}$ \\
\hline HB3 & $12.83 \pm 3.53 \mathrm{~h}$ & $33.50 \pm 3.23 \mathrm{e}$ \\
\hline HB4 & $16.50 \pm 3.89 \mathrm{~h}$ & $33.00 \pm 1.473 \mathrm{e}$ \\
\hline HB5 & $15.50 \pm 2.38 \mathrm{~h}$ & $28.33 \pm 3.04 \mathrm{e}$ \\
\hline HB6 & $15.17 \pm 2.05 \mathrm{~h}$ & $35.50 \pm 3.54 \mathrm{e}$ \\
\hline
\end{tabular}

${ }^{\mathrm{z}}$ Data in the table are the mean \pm standard error. Different letters indicate significant difference at $P<0.05$ level by Duncan's new multiple range test. electrophoresis results were observed with a gel-imaging system (Bio-raid 2200; Alpha Innotech Company, San Leandro, CA, U.S.A.) and photographed to record the experimental results.

The electrophoresis map obtained was analyzed using UVP Lab Works software, and the molecular weight of each electrophoresis band was automatically generated by using DL 5000 DNA (TaKaRa, Japan) as a marker. If there was an electrophoresis band at certain molecular weight, the assignment was " 1 " whereas, if there was no electrophoresis band, the assignment was " 0 ", producing a "0-1" table. The software of Ntsy-PC 1.8 (Execter Software, Setauket, NY, U.S.A.) (Rohlf 1993) was used for data analysis. In the SAHN classification program, the unweighted pair-group method with arithmetic means (UPGMA) was employed to automatically generate the similarity coefficients of each tested strain. Matrix and cluster analysis tree diagrams were performed.

\section{Results}

Differential analysis of pycnidium and conidium productions. Significant differences were detected in the amount of pycnidium and conidium production at the 0.05 level among the six provinces, including Jiangxi, Fujian, Hainan, Shandong, Shanxi, and Hebei. The amount of the pycnidia produced by the Fujian strains was relatively large, between 45 and 65 pycnidia/dish. Among them, FJ1 and FJ4 produced the highest amounts of pycnidia, with average productions at 62.50 and 60.17 pycnidia/dish, respectively. The amount of pycnidia produced by Hainan strains ranked the second, and was between 30 and 40 pycnidia/dish. The production of pycnidia in Jiangxi, Shanxi, Shandong, and Hebei Provinces was relatively low, between 10 and 20 pycnidia/dish. The conidium production of Fujian strains was relatively large, between $1.80 \times 10^{8}$ and $2.20 \times 10^{8}$ conidia/dish. The number of conidia produced by Hainan strain was the second, between $1.00 \times 10^{8}$ and $1.10 \times 10^{8}$ conidia/ dish, and the production of conidia in Jiangxi, Shanxi, Shandong, and Hebei Provinces was relatively low, between $25 \times 10^{6}$ and $4.00 \times 10^{7}$ conidia/dish (Table 4).

Analysis on difference of pathogenicity to different strains. Considerably different pathogenicity of asparagus stem fungus was established among the six provinces, with significantly different disease indexes at the 0.05 level. FJ 2 had the strongest pathogenic force, with a disease index of 75.33. HN2 and SD4 followed with disease indices of 74.07 and 73.88 , respectively. SD5 had the lowest disease index of 42.16 (Table 5).

Comparison of growth rate among different strains. Different colony diameters and weights of dried mycelia from Phomopsis asparagi strains were established. The colonies of HN1-5 were larger in diameter, between 8.43 and $8.61 \mathrm{~cm}$, followed by XT1-5, LT1-3, and FJ1-5, whose colony diameters were between 7.40 and $7.60 \mathrm{~cm}$. The colony diameter of SX6 was smallest, with a colony diameter at $4.34 \mathrm{~cm}$. The weight of dried mycelia from HN2 was largest, with a weight at $0.64 \mathrm{~g}$, followed by FJ1-5, HN1, and HN3-5, with weights of dried mycelia between 0.53 and $0.63 \mathrm{~g}$. The weight of dried mycelia from SX5 was lightest, with a weight at $0.26 \mathrm{~g}$ (Table 6).

ISSR genetic analysis. Four samples with high repeatability and specificity were selected from 100 ISSR primers. ISSR-PCR amplification was performed on $36 P$. asparagi strains, and a large number of clearly repeatable DNA fingerprints (amplified strips) were obtained. Most of the amplified fragments were within the range of 300 to $3,500 \mathrm{bp}$. A clear, bright, and repeatable band was obtained by each primer but the number of bands amplified was different (Fig. 2). In all, 69 total points, 64 multiple points, and 92.75\% polymorphism points were established. The number of ISSR gene sites detected by the four primers ranged from 14 to 20, with an average value of 16.00 multiple sites (Table 7).

According to the 0-1 table obtained by the amplification using the four ISSR primers, the similarity coefficient of Dice between the strains was calculated, followed by clustering using UPGMA method; then, the clustering diagram among $36 P$. asparagi strains and 1 Pyricularia oryzae strain was gained (Fig. 3). The highest similarity coefficient among the Phomopsis asparagi strains was 0.99 , while the lowest was 0.79 , which revealed that there existed larger genetic variation among strains and strains from different geographical sources clustered into different 
groups. When the similarity coefficient was approximately 0.86 , all $P$. asparagi strains clustered into three groups, while Pyricularia oryzae clustered to another single group. The composition of the three groups was as follows. The first group, named group I, contained six strains from Shandong, six strains from Shanxi, and six strains from Hebei, in which six strains from Shandong clustered to one single subgroup named subgroup I, six strains from Shanxi clustered to one single subgroup named subgroup II, and six strains from Hebei clustered to one single group named subgroup III. The second group, named group II, contained eight strains from Jiangxi and five strains from Fujian, in which eight strains from Jiangxi clustered to one single subgroup named subgroup IV and five strains from Fujian cluster to one single subgroup named subgroup V. The third group, named group III, contained five strains from Hainan, in which five strains from Hainan clustered into one single subgroup named subgroup VI. The results of cluster analysis showed that the strains from the neighboring provinces had a nearer phylogenetic relationship. The system evolution of asparagus stem blight fungus of Phomopsis asparagi was related to the geographical distribution of the strain.

Genotype diversity and genetic variation. The genetic diversity index $\mathrm{h}$ value of comprehensive Nei among the three groups is 0.2486 , ranging from 0.0603 to 0.0986 . The comprehensive value of Shannon index I is 0.3919 , ranging from 0.0853 to 0.1456 . The percentage of multiple-site points ranged from 0.0853 to 0.1456 , and the percentage of the comprehensive multiple-site points was $94.20 \%$. The h value, I value, numbers of multiple-site points, and the percentage of multiplesite points of group I were the largest of all the groups, which revealed that its genetic diversity was richer and its genetic variation was larger.

Table 5. Disease indexes of Phomopsis asparagi strains

\begin{tabular}{ll}
\hline Strains & \multicolumn{1}{c}{ Disease indexes } \\
\hline XT1 & $53.01 \pm 2.64$ milkjn \\
XT2 & $43.43 \pm 2.18$ mon \\
XT3 & $70.07 \pm 1.88$ bdac \\
XT4 & $48.66 \pm 3.25$ mlkojn \\
XT5 & $57.82 \pm 2.28$ eilkhgjf \\
LT1 & $55.03 \pm 3.35$ milkhgjn \\
LT2 & $66.93 \pm 3.89$ ebdacf \\
LT3 & $71.87 \pm 2.58$ bac \\
FJ1 & $64.75 \pm 2.08$ ebdagcf \\
FJ2 & $75.33 \pm 3.35$ a \\
FJ3 & $54.36 \pm 4.83$ milkhgjn \\
FJ4 & $46.29 \pm 1.18$ mon \\
FJ5 & $56.57 \pm 2.39$ milkhgjf \\
HN1 & $45.23 \pm 2.89$ on \\
HN2 & $74.07 \pm 4.27$ ba \\
HN3 & $62.17 \pm 3.44$ eidhgcf \\
HN4 & $57.71 \pm 3.74$ eilkhgjf \\
HN5 & $58.03 \pm 4.65$ eilkhgjf \\
SX1 & $60.47 \pm 1.13$ eidhgjf \\
SX2 & $49.65 \pm 1.98$ mlkojn \\
SX3 & $58.20 \pm 1.97$ eilkhgjf \\
SX4 & $47.41 \pm 2.14$ mlon \\
SX5 & $69.69 \pm 1.76$ bdac \\
SX6 & $64.27 \pm 2.14$ ebdhgcf \\
SD1 & $67.95 \pm 3.96$ ebdac \\
SD2 & $46.86 \pm 4.34$ mon \\
SD3 & $54.28 \pm 5.22$ milkhgjn \\
SD4 & $73.88 \pm 6.55$ ba \\
SD5 & $42.16 \pm 1.53$ o \\
SD6 & $60.58 \pm 2.33$ eidhgjf \\
HB1 & $50.45 \pm 1.95$ mlkojn \\
HB2 & $45.79 \pm 3.52$ mon \\
HB3 & $66.99 \pm 3.23$ bdac \\
HB4 & $59.42 \pm 1.47$ eidkhgjf \\
HB5 & $49.08 \pm 3.04$ mlkon \\
HB6 & $53.47 \pm 3.54$ milkhjn \\
\hline ID 19
\end{tabular}

${ }^{\mathrm{z}}$ Data in the table are the mean \pm standard error. Different letters indicate significant difference at $P<0.05$ level by Duncan's new multiple range test.
The genetic diversity of group I was richest, followed by group II, and that of group III was poorest (Table 8).

The genetic diversity index $h$ value of comprehensive Nei among the six subgroups was 0.3289 , ranging from 0.0093 to 0.0655 . The comprehensive value of Shannon index I was 0.4896, ranging from 0.0145 to 0.0879 . The percentage of multiple-site points ranged from 37.68 to $52.17 \%$, and the percentage of the comprehensive multiplesite points was $94.20 \%$. The $\mathrm{h}$ value, I value, numbers of multiple-site points, and the percentage of multiple-site points of subgroup VI from Hainan were the largest of all the groups, which revealed that its genetic diversity was richer and its genetic variation was larger. The genetic diversity of subgroup VI was the richest, followed by the central group, and that of subgroup VI from Hainan was the poorest (Table 9).

Analysis of genetic similarity. The similarity coefficient of six subgroups ranged from 0.3509 to 0.8832 and the related genetic distance ranged from 1.0471 to 0.1242 . The coefficient index between Shanxi subgroup and Hebei subgroup was the largest, with a coefficient at 0.8832 , which revealed that their phylogenetic relationship was the nearest. The coefficient index between Shanxi subgroup and Hainan subgroup was the lowest, with a coefficient at 0.3509 , which revealed that their phylogenetic relationship was the farthest (Table 10). The differences of similarity coefficients among populations were significant, which indicated that the genetic variation of $P$. asparagi strains from different geographical populations in China was large.

Group structure. The three groups of $P$. asparagi strains existed with genetic differentiation and the overall gene diversity index was 0.2372 . The average gene diversity among groups was 0.2177 , which proved that genetic variation existed mainly among groups and the

Table 6. Growth rates of Phomopsis asparagi strains $^{\mathrm{Z}}$

\begin{tabular}{|c|c|c|}
\hline Strains & Colony diameter (cm) & Dry weight of mycelia (g) \\
\hline XT1 & $7.48 \pm 0.05 b$ & $0.50 \pm 0.03 \mathrm{edfc}$ \\
\hline XT2 & $7.44 \pm 0.14 b$ & $0.51 \pm 0.03 \mathrm{ebdc}$ \\
\hline XT3 & $7.47 \pm 0.08 b$ & $0.48 \pm 0.03 \mathrm{egdf}$ \\
\hline XT4 & $7.44 \pm 0.08 b$ & $0.44 \pm 0.03$ egifh \\
\hline XT5 & $7.42 \pm 0.04 b$ & $0.50 \pm 0.03 \mathrm{edfc}$ \\
\hline LT1 & $7.45 \pm 0.05 b$ & $0.47 \pm 0.03$ egdfh \\
\hline LT2 & $7.40 \pm 0.10 b$ & $0.43 \pm 0.03$ egifhj \\
\hline LT3 & $7.43 \pm 0.05 b$ & $0.52 \pm 0.03$ ebdac \\
\hline $\mathrm{FJ} 1$ & $7.60 \pm 0.06 b$ & $0.62 \pm 0.04 \mathrm{bac}$ \\
\hline FJ2 & $7.53 \pm 0.08 b$ & $0.58 \pm 0.03 \mathrm{bdac}$ \\
\hline FJ3 & $7.50 \pm 0.13 b$ & $0.57 \pm 0.03$ bdac \\
\hline FJ4 & $7.44 \pm 0.09 b$ & $0.58 \pm 0.03$ bdac \\
\hline FJ5 & $7.52 \pm 0.09 b$ & $0.53 \pm 0.03$ ebdac \\
\hline HN1 & $8.61 \pm 0.06 \mathrm{a}$ & $0.63 \pm 0.03 \mathrm{ba}$ \\
\hline $\mathrm{HN} 2$ & $8.46 \pm 0.09 \mathrm{a}$ & $0.64 \pm 0.05 \mathrm{a}$ \\
\hline HN3 & $8.50 \pm 0.06 \mathrm{a}$ & $0.57 \pm 0.04$ bdac \\
\hline HN4 & $8.48 \pm 0.14 \mathrm{a}$ & $0.60 \pm 0.04 \mathrm{bac}$ \\
\hline HN5 & $8.43 \pm 0.12 \mathrm{a}$ & $0.61 \pm 0.04 \mathrm{bac}$ \\
\hline SX1 & $5.09 \pm 0.38 \mathrm{dc}$ & $0.33 \pm 0.03 \mathrm{kilj}$ \\
\hline SX2 & $5.13 \pm 0.35 \mathrm{dc}$ & $0.31 \pm 0.02 \mathrm{klj}$ \\
\hline SX3 & $4.88 \pm 0.20 \mathrm{de}$ & $0.28 \pm 0.02 \mathrm{kl}$ \\
\hline SX4 & $5.16 \pm 0.41 \mathrm{dc}$ & $0.30 \pm 0.04 \mathrm{kl}$ \\
\hline SX5 & $4.44 \pm 0.41 \mathrm{fe}$ & $0.26 \pm 0.031$ \\
\hline SX6 & $4.34 \pm 0.32 \mathrm{f}$ & $0.29 \pm 0.03 \mathrm{kl}$ \\
\hline SD1 & $5.39 \pm 0.07 \mathrm{dc}$ & $0.36 \pm 0.04$ kgilhj \\
\hline SD2 & $5.43 \pm 0.09 \mathrm{dc}$ & $0.38 \pm 0.03 \mathrm{kgilhj}$ \\
\hline SD3 & $5.33 \pm 0.04 \mathrm{dc}$ & $0.37 \pm 0.06$ kgilhj \\
\hline SD4 & $5.40 \pm 0.08 \mathrm{dc}$ & $0.37 \pm 0.04$ kgilhj \\
\hline SD5 & $5.25 \pm 0.05 \mathrm{dc}$ & $0.39 \pm 0.04 \mathrm{kgifhj}$ \\
\hline SD6 & $5.29 \pm 0.04 \mathrm{dc}$ & $0.39 \pm 0.04$ kgiflhj \\
\hline HB1 & $5.66 \pm 0.23 c$ & $0.34 \pm 0.03 \mathrm{kilj}$ \\
\hline HB2 & $5.46 \pm 0.07 \mathrm{c}$ & $0.35 \pm 0.03$ kilhj \\
\hline HB3 & $5.38 \pm 0.09 \mathrm{dc}$ & $0.37 \pm 0.04$ kgilhj \\
\hline HB4 & $5.47 \pm 0.06 \mathrm{c}$ & $0.36 \pm 0.06$ kgilhj \\
\hline HB5 & $5.58 \pm 0.08 c$ & $0.36 \pm 0.04$ kgilhj \\
\hline HB6 & $5.53 \pm 0.10 c$ & $0.39 \pm 0.05$ kgifhj \\
\hline
\end{tabular}

${ }^{\mathrm{z}}$ Data in the table are the mean \pm standard error. Different letters indicate significant difference at $P<0.05$ level by Duncan's new multiple range test. 
genetic variation within the group was relatively small. The overall genetic differentiation coefficient was 2.0299 and the gene flow was 1.2055, which confirms the existence of a larger gene flow among groups, and the genetic diversity was obvious among groups (Table 11).

\section{Discussion}

Pycnidium and conidium production is important for the identification and screening of resistance to asparagus stem blight (Yang et al. 2012a), and is also an important basis for morphological identification (Yang et al. 2012b, 2015a). In this study, we determined the

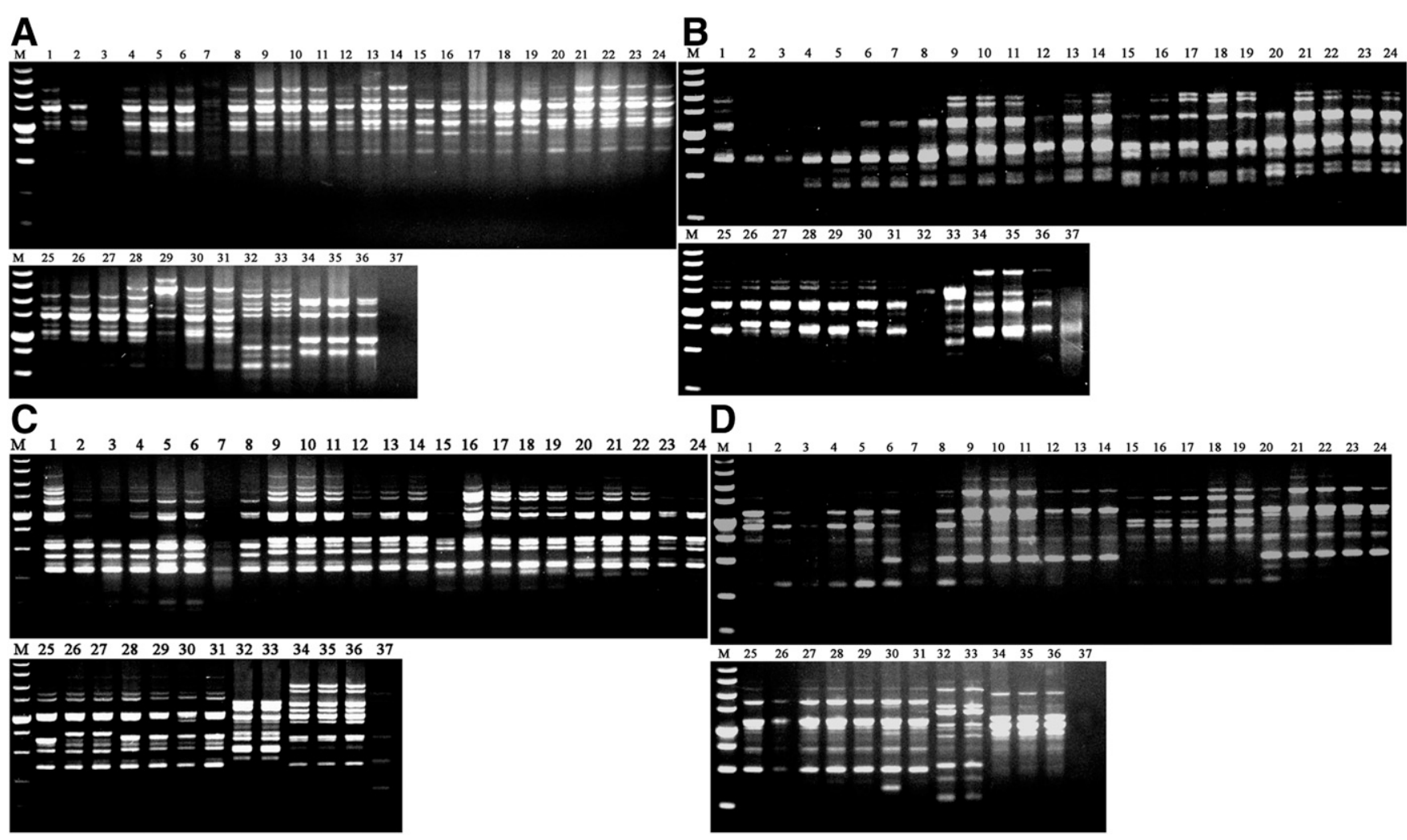

Fig. 2. Electrophoresis pattern of PCR-amplified products of genomic DNA of different isolates of Phomopsis asparagi. A, Primer 861; B, primer 888; C, primer 889; and D, primer 890. Lane M, DL5000 marker; lane 1, XT1; lane 2, XT2; lane 3, XT3; lane 4, XT4; lane 5, XT5; lane 6, LT1; lane 7, LT2; lane 8, LT3; lane 9, SD1; lane 10, SD2; lane 11, SD3; lane 12, SD4; lane 13, SD5; lane 14, SD6; lane 15, FJ1; lane 16, FJ2; lane 17, FJ3; lane 18, FJ4; lane 19, FJ5; lane 20, SX1; lane 21, SX2; lane 22, SX3; lane 23, SX4; lane 24, SX5; lane 25, SX6; lane 26, HB1; lane 27, HB2; lane 28, HB3; lane 29, HB4; lane 30, HB5; lane 31, HB6; lane 32, HN1; lane 33, HN2; lane 34, HN3; lane 35, HN4; lane 36, HN5; and lane 37, the outer fungus Pyricularia oryzae.

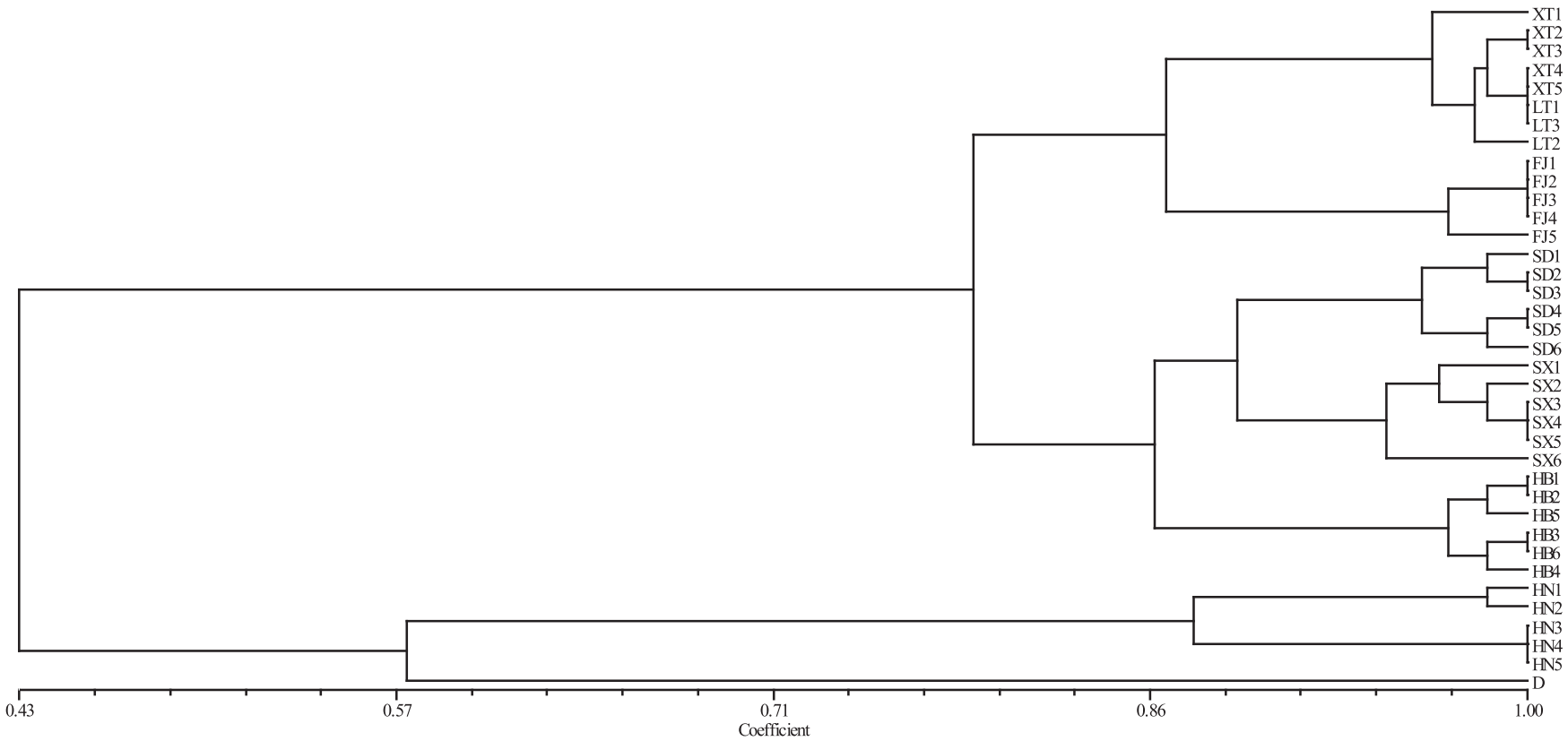

Fig. 3. Intersimple sequence repeat dendrogram of 36 isolates of Phomopsis asparagi . XT1-5, LT1-3, strains from Nanchang and Jiangxi; FJ1-5, strains from Fuzhou and Fujian; HN1-5, strains from Danzhou and Hainan; SX1-6, strains from Taiyuan and Shanxi; SD1-6, strains from Weifang and Shandong; HB1-6, strains from Baoding and Hebei; and D, the outer fungus Pyricularia oryzae. 
amount of pycnidium and conidium production of $P$. asparagi strains in six provinces. The effects of the culture medium, light, temperature, carbon, and nitrogen sources on the amount of pycnidium and conidium produced by $P$. asparagi strains were discussed in a previous study, and the change laws of the number of pycnidium and conidium under the conditions of gradient culture were clarified. The sporogenesis conditions of the pathogen were discussed earlier (Yang et al. 2015a). In this study, the optimum sporogenic culture conditions were used to elucidate the important differentiation of pycnidium and conidium in the provinces of asparagus stem blight pathogen. The results of the previous research were verified.

Resistance identification is the important avenue for gaining the resistant germplasms (Sonoda et al. 1997). The selection of appropriate strains for resistance screening in different asparagus germplasms is important for high efficiency and sensitivity (Yang et al. 2012a). Several inoculation methods were compared in the previous study, and the effectiveness and sensitivity of a spore-spraying method were established. The virulence of several strains of asparagus stem blight pathogen was determined, and the more virulent strains were selected as an inoculation stain for resistance identification (Yang et al. 2012a). On the basis of previous research findings, the spore-spray

Table 7. PCR amplification of genomic DNA of 36 strains of Phomopsis asparagi with intersimple sequence repeat primers

\begin{tabular}{lccc}
\hline Primer & Amplified band & Polymorphic band & Polymorphic rate (\%) \\
\hline 861 & 15 & 14 & 93.33 \\
888 & 15 & 14 & 93.33 \\
889 & 20 & 17 & 85.00 \\
890 & 19 & 19 & 100.00 \\
Average & 17.25 & 16.00 & 92.75 \\
Total & 69 & 64 & 92.75 \\
\hline
\end{tabular}

Table 8. Genotype diversity of Phomopsis asparagi populations from three groups $^{\mathrm{z}}$

\begin{tabular}{lcccccc}
\hline Population & na & ne & h & I & $\boldsymbol{N}_{\mathbf{P}}$ & $\boldsymbol{P ( \% )}$ \\
\hline Group I & 1.2609 & 1.1688 & 0.0986 & 0.1456 & 41 & 59.42 \\
Group II & 1.1884 & 1.1250 & 0.0703 & 0.1035 & 33 & 47.83 \\
Group III & 1.1304 & 1.1138 & 0.0603 & 0.0853 & 32 & 46.38 \\
Multiple populations & 1.8696 & 1.3862 & 0.2486 & 0.3919 & 65 & 94.20 \\
\hline
\end{tabular}

z Abbreviations: $\mathrm{na}=$ number of different alleles, $\mathrm{ne}=$ number of effective alleles, $\mathrm{h}=$ diversity index of Nei; $\mathrm{I}=$ index of Shannon; $N_{\mathrm{P}}=$ numbers of polymorphism sites; $P=$ percentage of polymorphism sites [(numbers of polymorphism sites/total numbers of sites $\times 100]$. Origin of group I $=$ Shandong, Shanxi, and Hebei from the north of China; origin of group II = Jiangxi and Fujian from the central area of China; and origin of group III = Hainan from the south of China.

Table 9. Genotype diversity of Phomopsis asparagi populations from six provinces $^{\mathrm{z}}$

\begin{tabular}{lcccccc}
\hline Population & na & ne & h & $\mathbf{I}$ & $\boldsymbol{N}_{\mathbf{P}}$ & $\boldsymbol{P ( \% )}$ \\
\hline Subgroup I & 1.0580 & 1.0401 & 0.0225 & 0.0332 & 31 & 44.93 \\
Subgroup II & 1.0870 & 1.0334 & 0.0242 & 0.0392 & 31 & 44.93 \\
Subgroup III & 1.0435 & 1.0317 & 0.0177 & 0.0258 & 36 & 52.17 \\
Subgroup IV & 1.0580 & 1.0209 & 0.0149 & 0.0245 & 30 & 43.48 \\
Subgroup V & 1.0290 & 1.0136 & 0.0093 & 0.0145 & 26 & 37.68 \\
Subgroup VI & 1.1304 & 1.1220 & 0.0655 & 0.0879 & 32 & 46.38 \\
Multiple populations & 1.9130 & 1.5712 & 0.3289 & 0.4896 & 65 & 94.20
\end{tabular}

z Abbreviations: $\mathrm{na}=$ number of different alleles, ne $=$ number of effective alleles, h $=$ diversity index of Nei, I = index of Shannon, $N_{\mathrm{P}}=$ numbers of polymorphism sites, and $P=$ percentage of polymorphism sites [(numbers of polymorphism sites/total numbers of sites) $\times 100$ ]. Origin of subgroup I = Shandong, origin of subgroup II = Shanxi, origin of subgroup III = Hebei, origin of subgroup IV = Jiangxi, origin of subgroup $\mathrm{V}=$ Fujian, and origin of subgroup VI = Hainan. method was used for inoculation, which further clarified the difference in pathogenic force of strains in Jiangxi and other provinces. In this investigation, the seven-grade classification standard of asparagus stem blight was established. Compared with the four-level classification standard made by Yang et al. (2012a) and Sonoda et al. (1997), differences in the virulence among strains were more obvious, which is more conducive to the determination of the degree of virulence differentiation of $P$. asparagi strains from different provinces.

Liu et al. (1994) found that the mycelial growth rate of Shandong, Jiangsu, Jiangxi, and Hubei strains was faster, while that of Fujian and Zhejiang strains was slower. Yang et al. (2012b) found that Hainan strains grew the fastest, followed by Fujian and Jiangxi strains, and Shandong and Hebei strains grew more slowly; that is, the strains in southern China grew faster than those in northern China. We found that Hainan strains grew the fastest, followed by those from Fujian and Jiangxi strains, and Shanxi, Shandong, and Hebei strains grew slowly, which confirmed that asparagus stem blight fungus possessed obvious differentiation among provinces and regions, which supported the research results of Yang et al. (2012b).

The diversity of plant fungi was studied using the ISSR marking method by some researchers (Karwasra et al. 2002; Sharma et al. 2005; Stodart et al. 2007). In this article, the diversity of $P$. asparagi strains from Jiangxi, Fujian, Hebei, Shandong, Shanxi, and Hainan Provinces was studied using the ISSR marking method and the results revealed that the strains of the neighboring provinces had a nearer phylogenetic relationship. The results obtained were in agreement with the diversity results based on rDNA ITS (Yang et al. 2012b).

It has been proved that strains from different climatic zones express obvious regional differentiation, while strains from the same climatic zone show similar biological and genetic characteristics. The pathogens of asparagus stem blight from different climatic zones are influenced by environmental factors, especially temperature. Therefore, biological characteristics and genetic diversity change with the geographic location. Growth of $P$. asparagi requires suitable temperatures (Yang et al. 2015a). In the tropical zone, temperature can remain within a suitable range and the pathogens can grow well all the year. However, in the northern temperate zone, the lowest temperature could be below $0^{\circ}$ in winter; therefore, pathogens have to adapt to the low temperature, and the biological and genetic characteristics have

Table 10. Coefficients of Nei's genetic comparability and genetic distances ${ }^{z}$

\begin{tabular}{lcccccc}
\hline Locations & Jiangxi & Shandong & Fujian & Shanxi & Hebei & Hainan \\
\hline Jiangxi & $\ldots$ & 0.8548 & 0.8769 & 0.5525 & 0.5212 & 0.4958 \\
Shandong & 0.1569 & $\ldots$ & 0.8198 & 0.6777 & 0.6212 & 0.4125 \\
Fujian & 0.1313 & 0.1987 & $\ldots$ & 0.5837 & 0.5485 & 0.5573 \\
Shanxi & 0.5933 & 0.3891 & 0.5384 & $\ldots$ & 0.8832 & 0.4199 \\
Hebei & 0.6516 & 0.4761 & 0.6006 & 0.1242 & $\ldots$ & 0.3509 \\
Hainan & 0.7015 & 0.8856 & 0.5847 & 0.8678 & 1.0471 & $\ldots$ \\
\hline
\end{tabular}

${ }^{\mathrm{z}}$ The upper section is the genetic distance between groups and the lower section is the coefficient of similarity between groups.

Table 11. Genetic diversity, gene differentiation, and their coefficients for three populations of Phomopsis asparagi from six provinces of China ${ }^{\mathrm{z}}$

\begin{tabular}{lccccc}
\hline Population & Population size & $\boldsymbol{H}_{\mathbf{T}}$ & $\boldsymbol{H}_{\mathbf{S}}$ & $\boldsymbol{G}_{\mathbf{S T}}$ & $\boldsymbol{N} \mathbf{m}$ \\
\hline Northern group & 18 & 0.1998 & 0.0231 & 0.8844 & 0.0654 \\
Central group & 13 & 0.0752 & 0.0136 & 0.8185 & 0.1109 \\
Southern group & 5 & 0.0432 & 0.0291 & 0.3270 & 1.0292 \\
Average & 12 & 0.1061 & 0.0219 & 0.6766 & 0.4018 \\
Overall & 36 & 0.3182 & 0.0658 & 2.0299 & 1.2055
\end{tabular}

${ }^{\mathrm{z}}$ Abbreviations: $H_{\mathrm{T}}=$ gene diversity index of total groups, $H_{\mathrm{S}}=$ gene diversity index within subgroups, $G_{\mathrm{ST}}=$ genetic differentiation coefficient, and $N \mathrm{~m}=$ gene flow . 
changed in the long course of evolution. Thus, the biological and genetic characteristics of $P$. asparagi strains from different geographic locations differ because of their long-term evolution under different environmental factors, especially the temperature.

The biological characteristics and genetic diversity of asparagus stem blight fungi may be due to the differences in geographical environment and climate conditions of the various regions. In the long-term coevolution process with the host, the continuous exchange of genetic information within the population has led to the maintenance of relatively independent genetic diversity in different regions. Understanding the pathogenicity differentiation of strains in different regions is helpful to guide farmers from different geographical regions to strengthen the control strategy of the disease, adjust the planting system, and provide the theoretical basis for molecular breeding for resistance to asparagus stem blight and disease prediction and prevention.

\section{Literature Cited}

Chen, G. Y. 2005. Pages 7-11 in: Technology of Pollution-Free Production of Asparagus. China Agriculture Press, Beijing, China.

Davis, R. D. 2001. Asparagus stem blight recorded in Australia. Australas. Plant Pathol. 30:181-182.

Elena, K. 2006. First report of Phomopsis asparagi causing stem blight of asparagus in Greece. Plant Pathol. 55:300.

Fang, Z. D. 1998. Pages 46-50 in: Research Methods of Plant Disease, third ed. China Agriculture Press, Beijing, China.

Guleria, S., Aggarwal, R., Thind, T. S., and Sharma, T. R. 2007. Morphological and pathological variability in rice isolates of Rhizoctonia solani and molecular analysis of their genetic variability. J. Phytopathol. 155:654-661.

Guo, M., Bian, Y., Wang, J., Wang, G., Ma, X., and Xu, Z. 2017. Biological and molecular characteristics of a novel partitivirus infecting the edible fungus Lentinula edodes. Plant Dis. 101:726-733.

Karwasra, S. S., Mukherjee, A. K., Swain, S. C., Mohapatra, T., and Sharma, R. P. 2002. Evaluation of RAPD, ISSR and AFLP markers for characterization of the loose smut fungus Ustilago tritici. J. Plant Biochem. Biotechnol. 11:99-103.

Liu, K. J. 2001. Pages 38-42 in: Practical Technology for High-Yield Cultivation of Asparagus. China Agriculture Press, Beijing, China.

Liu, K. J., Lu, Y. J., Chen, Y. X., and Li, S. J. 1994. Biological characters of Phomopsis asparagi (Sacc.) Bubak. Acta Phytopathol. Sin. 24:299-304.

Liu, K. J., Zhang, F. R., and Chen, Y. C. 1991. Studies on pathogen of stem blight of asparagus officinalis. Acta Mycol. Sin. 10:329-330.
Liu, Z. H., Sun, J., Yang, H., Mu, L. S., and Zhang, R. 2008. Biologica characteristics of Phomopsis asparagi the pathogen of asparagus stem blight. J. Shenyang Agric. Univ. 39:301-304.

Meng, F., Yang, Y. Q., Lan, B., and Li, X. M. 2013. Difference of fungicide resistance of asparagus stem blight fungus from different geographic origins. J. Huazhong Agric. Univ. 32:61-65.

Miao, H. M., Hou, X. Y., Meng, F. M., and Xu, Y. H. 1991. Biological characteristics of the pathogenic fungi of stem blight of asparagus. Acta Phytophylac. Sin. 18:87-90.

Reddy, M. P., Sarla, N., and Siddiq, E. A. 2002. Inter simple sequence repeat (ISSR) polymorphism and its application in plant breeding. Euphytica 128:9-17.

Rosewich, U. L., Pettway, R. E., McDonald, B. A., and Kistler, H. C. 1999. High levels of gene flow and heterozygote excess characterize Rhizoctonia solani AG-1 IA (Thanatephorus cucumeris) from Texas. Fungal Genet. Biol. 28: 148-159.

Sharma, M., Gupta, S. K., and Sharma, T. R. 2005. Characterization of variability in Rhizoctonia solani by using morphological and molecular markers. J. Phytopathol. 153:449-456.

Sonoda, T., Uragami, A., and Kaji, K. 1997. Evaluation of Asparagus officinalis cultivars for resistance to stem blight by using a novel inoculation method. HortScience 32:1085-1086.

Stodart, B. J., Harvey, P. R., Neate, S. M., Melanson, D. L., and Scott, E. S. 2007. Genetic variation and pathogenicity of anastomosis group 2 isolates of Rhizoctonia solani in Australia. Mycol. Res. 111:891-900.

Uecker, F. A., and Johnson, D. A. 1991. Morphology and taxonomy of species of Phomopsis on Asparagus. Mycologia 83:192-199.

Yang, Y. Q., Lan, B., Hu, S. X., Chang, D. D., Zhang, S. L., and Li, X. M. 2015a Effects of several factors on the production of pycnidia and conidia in asparagus stem blight pathogen. J. Plant Prot. 42:517-522.

Yang, Y. Q., Lan, B., Jian, Y. L., Chang, D. D., Zhang, S. L., and Li, X. M. 2016. Infection process and pathogenic mechanism of Phomopsis asparagi, the asparagus stem blight pathogen. Phytoparasitica 44:11-18.

Yang, Y. Q., Lan, B., Jian, Y. L., Hu, S. X., Chang, D. D., Zhang, S. L., and Li, X. M. 2015b. Establishment of Agrobacterium tumefaciens-mediated transformation system for Phomopsis asparagi, the pathogen of asparagus stem blight. Turk. J. Biochem. 40:290-297.

Yang, Y. Q., Lan, B., Sun, Q., Chen, H. F., Chen, J., Yu, J., and Li, X. M. 2018 Control effects of six fungicides on asparagus stem blight disease. Plant Prot. 44:217-220.

Yang, Y. Q., Li, X. M., Meng, F., Lan, B., and Zhang, J. 2012a. Establishment of a resistance-identification method on asparagus stem blight and screening of resistant asparagus germplasm resources. Acta Phytopathol. Sin. 42:649-654.

Yang, Y. Q., Li, X. M., Meng, F., Lan, B., and Zhang, J. 2012b. Identification of the pathogen of asparagus stem blight and analysis of its regional difference. $J$ Plant Prot. 39:315-320. 